\title{
金属间化合物 $\mathrm{LaFe}_{y} \mathrm{Ni}_{5_{-y}}$ Mössbauer 效应的研究
}

\author{
戴守思 孙 序* 王金玲 王朝果 \\ (中国科学院物理研究所, 北京)
}

$\mathrm{RB}_{5}$ 型桸土与过渡族金属间化合物，是优良的磁性材料与贮氢材料,对它们进行研究具有 重要的意义. $\mathrm{LaFeNi}_{4}$ 是其中之一, 它具有优良的氢海绵特性. Van Mal、三沢俊平 ${ }^{[1]}$ 等曾 对它进行过研究, 我们曾用不同的活化处理工艺, 获得了与他们不同的结果 ${ }^{[2]}$. 对于 LaNis 型材料过去人们用希土源进行过 Mőssbauer 效应的研究 ${ }^{[3]}$,但是用常见的 ${ }^{57} \mathrm{Co}$ 源对它们进行 Mössbauer 效应的研究尚少有报道. 下面我们探索用 ${ }^{~} \mathrm{Co}$ 源对 $\mathrm{LaFe}_{y} \mathrm{Ni}_{i_{-y}}$ 进行 Mössbauer 效 应的研究, 并辅以 $\mathrm{X}$ 射线结构分析.

\section{一、材料的制备}

我们使用纯度为 $99 \%$ 的 $\mathrm{La}, 99.9 \%$ 的 $\mathrm{Ni}$ 和 $99.99 \%$ 的 $\mathrm{Fe}$ 的纯金属作为原材料, 按其化 学组份要求眍制,在充 $\mathrm{Ar}$ 的真空电弧炉内熔化, 经过 5-6 次反复熔化淬冷, 制成组份均匀的 合金.

\section{二、实䏩结果与讨论}

1. X 射线结构分析 实验是在 RU-1000 型转虽 X 射线机进行的. 样品粉碎后经 350 目篮网过篮，用 Co 妿进行衍射测量; $2 \theta$ 角测量精度为 $0.02^{\circ}$ ，晶格参数测量精度为 $0.001 \AA$. 实验表明, $\mathrm{LaFe}_{y} \mathrm{Ni}_{5-y}$ 为 $\mathrm{CaCu}_{5}$ 型结构, 晶格参数为 $y$ 的函数,其数值见表 1 .

表 $1 \mathrm{LaFe}_{2} \mathrm{Ni}_{5-}$, 在室温的晶格参数

\begin{tabular}{c|c|c|c|c|c|c|c|c}
\hline$y$ & 0 & 0.5 & 0.6 & 0.7 & 0.8 & 0.9 & 1 \\
\hline$a(\AA)$ & 5.002 & 5.032 & 5.032 & 5.034 & 5.042 & 5.048 & 5.050 \\
$C(\AA)$ & 3.969 & 3.968 & 3.980 & 4.010 & 4.011 & 4.014 & 4.017 \\
$V\left(\AA^{3}\right)$ & 86.01 & 87.01 & 87.27 & 88.00 & 88.30 & 88.58 & 88.72 \\
\hline
\end{tabular}

表 $2 \mathrm{LaNi}$, 在温度 $10-300 \mathrm{~K}$ 的晶格参数

\begin{tabular}{c|c|c|c|c}
\hline$T(K)$ & 300 & 150 & 75 & 10 \\
\hline$a(\AA)$ & 5.002 & 4.989 & 4.988 & 4.985 \\
$C(\AA)$ & 3.969 & 3.965 & 3.965 & 4.965 \\
$V\left(\AA^{3}\right)$ & 86.01 & 85.46 & 85.43 & 85.33 \\
\hline
\end{tabular}

本文 1982 年 8 月 30 日收到.

*冶金部有色金属研究总院。 
在温度 $10-300 \mathrm{~K}$ 间, $\mathrm{LaNi}_{3}$ 的晶格参数见表 2, 低温下,它的晶格参数几乎与温度无关.

2. 室温 Mössbauer 效应的研究 我们使用 Elscint 公司的 Mössbauer 谱仪研究不同 $y$ 值 的 $\mathrm{LaFe}_{y} \mathrm{Ni}_{5-y}$ 的 Mössbauer 效应. 实验条件如下: 放射源为 ${ }^{57} \mathrm{Co} / \mathrm{Rh}$, 强度为 $50 \mathrm{mci}$, 样品 物理厚度为 3-4mg/ $\mathrm{cm}^{2}$, 每道计数率大于 $2 \times 10^{6}$. 实验结果用 Lorentz 线型和曼小二乘法 拟合, 拟合精度为 $0.001, \chi$ 平方值约为 $300-400$. $\mathrm{LaFe}_{y} \mathrm{Ni}_{5-y}$ 的室温的 Móssbauer 谱见图 1 ，拟合后的 Mössbauer 参数见表 3. 表中 $\delta_{I \cdot S}$ 为谱中心相对于金属铁的能量位移（它 为同质异能位移 $S_{l . s}$ 和二级 Dopple 位移之 和,现在 $S_{S O D}$ 可看为常数), $\Delta E_{Q . S}$ 为四极 分裂间距, FWHM 为半高峰宽, $A / A_{0}$ 为相 对面积比， $f$ 因子为无反冲因子.

首先, 由图 1 可以看到, 室温 Mössbauer 共振吸收谱具有明显的不对称性, 拟合结果 说明它由两组四极分裂谱组成. $X$ 射线衍射 分析已证明它们是单相材料, 这两组谱只能 对应于不同的晶位，一组约占总面积的 $90 \%$ ， 一组约占面积的 $10 \%$. Percheron-Guegon 等 人 ${ }^{[4]}$ 对 $\mathrm{LaMnNi}_{4}$ 所作的中子衍射研究表明, 大部分 $\mathrm{Mn}$ 原子位于 $3 \mathrm{~g}$ 位, 余下部份占有 $2 C$ 位. 我们认为 $\mathrm{Fe}$ 原子的分布与 $\mathrm{Mn}$ 原子的 相似, 约 $90 \%$ 的 $\mathrm{Fe}$ 原子占有 $3 \mathrm{~g}$ 位, 余下的 约 $10 \%$ 占有 $2 C$ 位.

其次,由表 3 可以看到, $\Delta E_{Q .5}$ 为 $y$ 的函 数, $3 \mathrm{~g}$ 位和 $2 C$ 位数值道从着不同的变化规 律,大体上讲, $3 \mathrm{~g}$ 位的 $\Delta E_{Q . s}$ 随 $y$ 值的增大 而减少, $2 C$ 位的 $\Delta E_{Q . S}$ 随 $y$ 值的增大而增

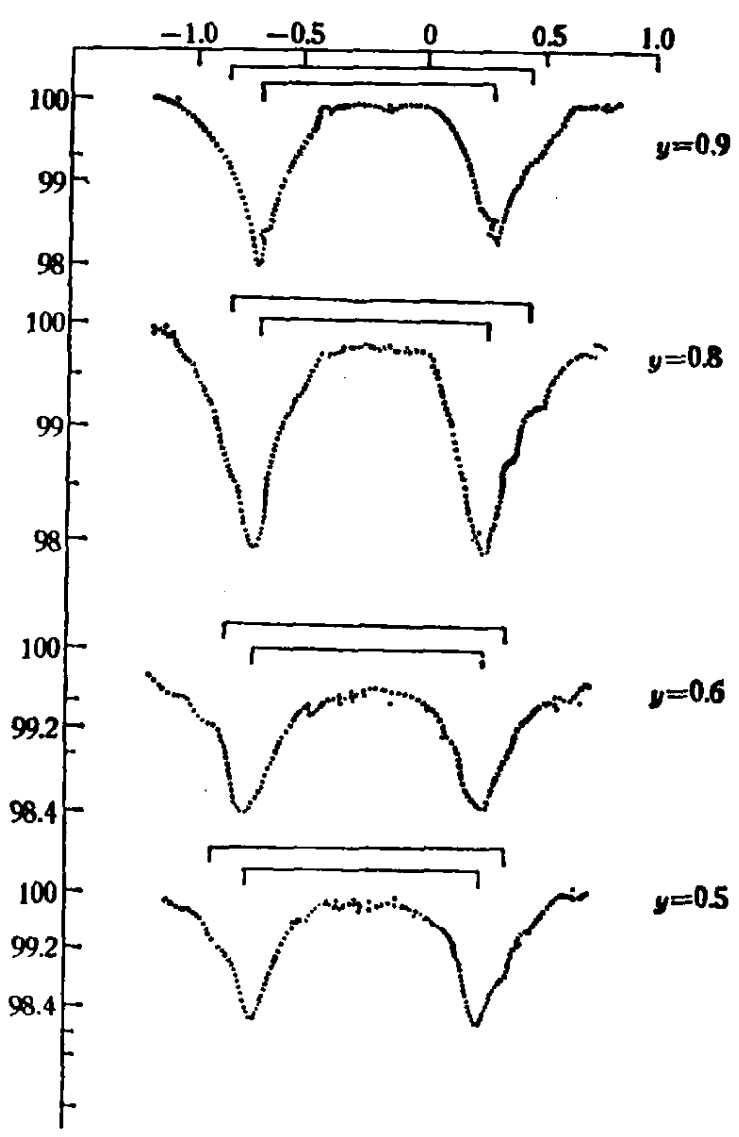

图 $1 \mathrm{LaFe}_{2} \mathrm{Ni}_{3 \rightarrow}$, 的室温 Mössbauer 服收渞 放射硕 ${ }^{37} \mathrm{Co} / \mathrm{Rh}$

大. 从 $\mathrm{X}$ 射线分析,已知 $\mathrm{LaFe}_{y} \mathrm{Ni}_{5-y}$ 为 $\mathrm{CaCu}_{5}$ 型结构, $3 \mathrm{~g}$ 位的 $\mathrm{Fe}$ 原子位于一变形的八面体 内 $[z=1 / 2$ 平面内四个 $\mathrm{Ni}(\mathrm{Fe})$ 原子 $(3 g), z=0$ 和 $z=1$ 平面内各两个 $\mathrm{Ni}(\mathrm{Fe})$ 原子 表 $3 \mathrm{LaFe}_{y} \mathrm{Ni}_{y_{-},}$的室温 Mössbauer 谱数数

\begin{tabular}{|c|c|c|c|c|c|}
\hline$y$ & $\frac{\delta_{I} \cdot \mathrm{s}}{\mathrm{mm} \cdot \mathrm{s}^{-1}}$ & $\frac{\Delta E_{Q \cdot s}}{\mathrm{~mm} \cdot \mathrm{s}^{-1}}$ & $\begin{array}{l}\text { FWHM } \\
\mathrm{mm} \cdot \mathrm{s}^{-1}\end{array}$ & $A / A_{0}$ & $t$-因子 \\
\hline 0.5 & $\begin{array}{l}-0.110 \\
-0.142\end{array}$ & $\begin{array}{l}0.985 \\
1.252\end{array}$ & $\begin{array}{l}0.246 \\
0.260\end{array}$ & $\begin{array}{l}0.91 \\
0.09\end{array}$ & 0.032 \\
\hline 0.6 & $\begin{array}{l}-0.113 \\
-0.134\end{array}$ & $\begin{array}{l}0.984 \\
1.257\end{array}$ & $\begin{array}{l}0.262 \\
0.268\end{array}$ & $\begin{array}{l}0.92 \\
0.08\end{array}$ & 0.033 \\
\hline 0.7 & $\begin{array}{l}-0.111 \\
-0.076\end{array}$ & $\begin{array}{l}0.979 \\
1.311\end{array}$ & $\begin{array}{l}0.258 \\
0.252\end{array}$ & $\begin{array}{l}0.92 \\
0.08\end{array}$ & 0.037 \\
\hline 0.8 & $\begin{array}{l}-0.107 \\
-0.080\end{array}$ & $\begin{array}{l}0.977 \\
1.275\end{array}$ & $\begin{array}{l}0.256 \\
0.228\end{array}$ & $\begin{array}{l}0.89 \\
0.11\end{array}$ & 0.043 \\
\hline 0.9 & $\begin{array}{l}-0.105 \\
-0.089\end{array}$ & $\begin{array}{l}0.980 \\
1.317\end{array}$ & $\begin{array}{l}0.268 \\
0.250\end{array}$ & $\begin{array}{l}0.93 \\
0.07\end{array}$ & 0.047 \\
\hline
\end{tabular}




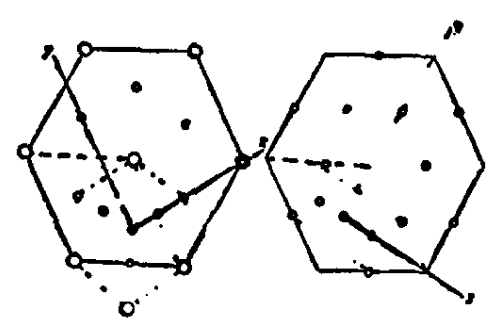

国 $2 \mathrm{LaFC}_{3} \mathrm{Ni}_{3-}$, 的结构和电场梯度计筆中 所选用的坐标系

O : La 原于； 0 为本平面及上下平面内的 $\mathrm{Ni}(\mathrm{Fe})$ 原于

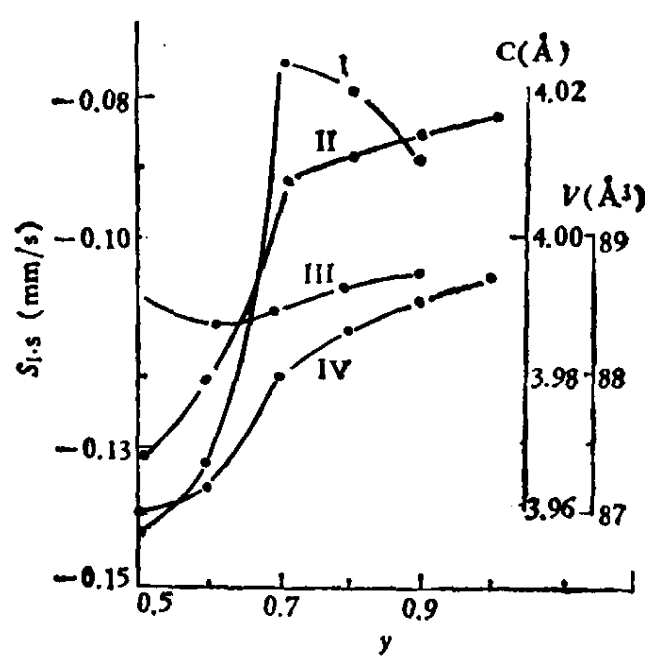

图 $3 \mathrm{LaFe}_{\mathrm{N}} \mathrm{Ni}_{3,-,}$ 的同质异能位移, $S_{1,3}$ 晶胞 体积 $(V) C$ 轴长度与 $y$ 的关系

1. $2 C$ 位 $\mathrm{Fe}$ 原子的 $\mathrm{S}_{I, s} ; 11$, 晶狍 $C$ 牰长度；

III. $3 g$ 位 $\mathrm{Fe}$ 原子的 $S_{l, s}$; IV. 晶軳体积

(2C) 作为它的顶点]，2C 位的 $\mathrm{Fe}$ 原子位于 20 面体内（z-1 平面内三个 $\mathrm{La}$ 原子和三个 $\mathrm{Ni}(\mathrm{Fe})$ 原子 $(2 C), z=1 / 2$ 和 $z-3 / 2$ 平面内各三个 $\mathrm{Ni}(\mathrm{Fe})$ 原子 ( $3 g$ ) 作为它的顶点). 若我们假定配位体是电荷为 $\boldsymbol{z}$ 的点电荷, 选择适当坐标系 (图 2), 可以算出配位体电荷在 Móssbauer 核处所产生的电场梯度张量. 对于 $3 g$ 位 $\mathrm{Fe}$ 原子

$$
\begin{array}{ll}
V_{x y}-4.25 z_{2 c} e e_{2 c}^{-3}-4 z_{3 g} e r_{38}^{-3}, & V_{x x}=-0.25 z_{2 c} \operatorname{er} 2 c+2 z_{3 g} e r_{38}^{-3}, \\
V_{y y}=-4 z_{2 c} e r_{2 c}^{-3}+2 z_{3 g} e r_{38}^{-3}, & V_{x y}=V_{y x}=V_{x z}=V_{z x}=V_{x y}=V_{y z}=0 .
\end{array}
$$

对于 $2 C$ 位 $\mathrm{Fe}$ 原子

$$
\begin{aligned}
& V_{x s}-6.37 z_{3 g} \operatorname{er}_{38}^{-3}-3 \operatorname{er}_{2 C}^{-3}\left[z_{L s}+Z_{N i}\right] \text {, } \\
& V_{x x}=-4.30 z_{3 g} \operatorname{er}_{3 g}^{-3}+1.5 \operatorname{er}_{2 C}^{-3}\left[z_{\mathrm{La}}+z_{\mathrm{Ni}}\right] \text {, } \\
& V_{y y}=-3.19 z_{3 g} e_{38}^{-3}+1.5 \operatorname{cr}_{2 c}^{-3}\left[z_{\mathrm{Lz}}+z_{\mathrm{Ni}}\right] \text {, } \\
& V_{x y}=V_{y x}-V_{x x}=V_{x x}=V_{y z}=V_{x y}=0 \text {. }
\end{aligned}
$$

我们巳知: $\mathrm{Ni}$ 一般为 $\mathrm{Ni}^{2+}$ 态, 而从 Mössbauer 参数看, $\mathrm{Fe}$ 为 $\mathrm{Fe}^{3+}$ 态, 因此 $z(\mathrm{Fe})>$ $z(\mathrm{Ni})$. 当 $\mathrm{Fe}$ 原子替代 $3 g$ 位 (几率为 $90 \%$ ) 的 $\mathrm{Ni}$ 原子, 配位体电荷 $z_{3 g}$ 的变化,将使 $3 g$ 位 ${ }^{57} \mathrm{Fe}$ 核处的 $V_{z x}$ 变小, $2 C$ 位 ${ }^{57} \mathrm{Fe}$ 核处的 $V_{\mathrm{s}}$ 变大; 由于 $\Delta E_{Q .5}$ 与 $V_{\mathrm{sz}}$ 成比例,因此 $3 g$ 位 的 $\Delta E_{Q . s}$ 随 $y$ 的增大而减少, $2 C$ 位的 $\Delta E_{Q . s}$ 随 $y$ 的增大而增大.

第三,由表 3 我们看到, $\mathrm{LaFe}_{y} \mathrm{Ni}_{5-y}$ 的同质异能位移是 $y$ 的函数,但 $3 \mathrm{~g}$ 位和 $2 C$ 位的数值 道从不同变化规律, 其实验曲线见图 3. 由大量实验事实已知 ${ }^{[5]}$, 一些化合物内 $\mathrm{Fe}$ 原子的同 质异能位移随原子间距增大而增大, 随近邻原子电负性的下降而减少. 在 $\mathrm{LaFe}_{y} \mathrm{Ni}_{3-y}$ 内, 这 两个矛盾因意使不同晶位的 $\delta_{1 \cdot s}$ 道从不同的变化规律; 当 $\mathrm{Fe}$ 取代 $\mathrm{Ni}$, 晶格膨胀, 同质异能位 移相应增大, 它与实验数值的差值, 我们假定为电负性下降的贡献. 由高压实验已知 ${ }^{[6]}, \mathrm{Ni}-$ $\mathrm{Fe}$ 合金的 $\Delta \delta_{1.5}$ 和 $\frac{\Delta V}{V}$ ( $V$ 为体积)的比值为 1 ; 依据 $3 \mathrm{~g}$ 位 $\delta_{I .5}$ 的数值, 用此估计电负性的 贡献是 $-0.06 \leqslant \frac{\Delta \delta_{1.5}}{\Delta y} \leqslant-0.03$. 此外, 因大部分 $F e$ 原子分布于 $3 g$ 位, 影响 $2 C$ 位 $\delta_{t . s}$ 的结构因意是 $C$ 轴的增大, 若考虑上述电负性下降的影响, 由 $2 C$ 位实验值得出 $1.75 \leqslant$ 
$\frac{\Delta \delta_{l . S}}{\Delta C} \leqslant 1.90$, 此数值略大于 $\mathrm{Fe}^{3+}-\mathrm{O}^{2-}$ 系列的实验值 ${ }^{[9]}$.

依据 Bhide 的公式 ${ }^{[7]}$,计算了 $\mathrm{LaFe}_{y} \mathrm{Ni}_{g_{-} y}$ 的 $f$ 因子(表 3)，结果表明, $f$ 因子随 $y$ 增大而 增大.

表 4 温度 $77-300 \mathrm{~K}$ 间 $\mathrm{LaFe}_{0 .}, \mathrm{Ni}_{4.3}$ 的 Mössbauer 参数

\begin{tabular}{|c|c|c|c|c|c|}
\hline$T(K)$ & $\underset{\mathrm{mm} \cdot \mathrm{s}^{-1}}{\delta_{1 \cdot \mathrm{s}}}$ & $\underset{\mathrm{mm} \cdot \mathrm{s}^{-1}}{\Delta E_{\mathrm{Q}} \cdot \mathrm{S}}$ & $\begin{array}{l}\text { FWMHI } \\
\mathrm{mm} \cdot \mathrm{s}^{-1}\end{array}$ & $A / A_{0}$ & $\underset{\mathrm{mm} \cdot \mathrm{s}^{-1}}{S_{Y}}$ \\
\hline 300 & $\begin{array}{l}-0.111 \\
-0.076\end{array}$ & $\begin{array}{l}0.979 \\
1.311\end{array}$ & $\begin{array}{l}0.258 \\
0.252\end{array}$ & $\begin{array}{l}0.92 \\
0.08\end{array}$ & $\begin{array}{l}5.495 \\
5.530\end{array}$ \\
\hline 200 & $\begin{array}{l}-0.048 \\
-0.026\end{array}$ & $\begin{array}{l}0.978 \\
1.318\end{array}$ & $\begin{array}{l}0.304 \\
0.256\end{array}$ & $\begin{array}{l}0.92 \\
0.08\end{array}$ & $\begin{array}{l}1.135 \\
1.157\end{array}$ \\
\hline 137 & $\begin{array}{r}-0.016 \\
0.025\end{array}$ & $\begin{array}{l}1.021 \\
1.536\end{array}$ & $\begin{array}{l}0.342 \\
0.276\end{array}$ & $\begin{array}{l}0.93 \\
0.07\end{array}$ & $\begin{array}{l}0.318 \\
0.359\end{array}$ \\
\hline 77 & $\begin{array}{l}0.009 \\
0.086\end{array}$ & $\begin{array}{l}1.066 \\
1.558\end{array}$ & $\begin{array}{l}0.354 \\
0.386\end{array}$ & $\begin{array}{l}0.93 \\
0.07\end{array}$ & $\begin{array}{l}0.127 \\
0.204\end{array}$ \\
\hline
\end{tabular}

\section{三、不同週度 Mössbauer 效应研究}

我们在温度 $77-300 \mathrm{~K}$ 间对 $\mathrm{LaFe}_{0.7} \mathrm{Ni}_{4.3}$ 进行了 Mössbauer 效应的研究, 控温精度为 $0.01^{\circ}$. 其共振吸收谱见图 4, 它们的 Mössbauer 参数见表 4 .

首先,由表 4 看出, 随温度下降, 四极分 裂间距 $\Delta E_{Q .5}$ 逐渐增大. 现配位体电荷无改 变, ${ }^{57} \mathrm{Fe}$ 核处电场梯度的变化只能来自于原 子本身内 $3 d$ 电子分布的变化, 这可能是由于 $3 d$ 带内热电子分布遵从 Boltzmann 统计 ${ }^{[8]}$. 其次， $\delta_{1 \cdot s}$ 随温度的下降而增大，基于固体 的 Debye 模型, 以类似 Wertheim 的方式 ${ }^{[0]}$, 由能量位移 $\delta_{1.5}$ 与温度的关系, 估算 $\mathrm{LaFe}_{0.7}$ $\mathrm{Ni}_{4,3}$ 的 Debye 温度为 $346 \mathrm{~K}$. 第三, 我们 依据 Debye 模型,计算了二级 Dopple 位移 $S_{S O D}$, 由 $\delta_{I . S}$ 和 $S_{S O D}$ 之差, 得出经热运动修 正后的同质异能位移 $S_{t . s}$; 结果表明, $\mathrm{LaFe}_{0.7}$ $\mathrm{Ni}_{4.3}$ 的 $S_{I . S}$ 随温度的下降而减少,此规律与 自然铁及 $\mathrm{Cu}-\mathrm{Fe}$ 系统相 似 ${ }^{[10 \mathrm{e}]}$, 对此现象人 们曾进行过讨论, 但尚未得到满意的解释.

\section{四、结 语}

实验证明, $\mathrm{LaFe}_{v} \mathrm{Ni}_{5-y}$ 的结构为 $\mathrm{CaCu}_{5}$

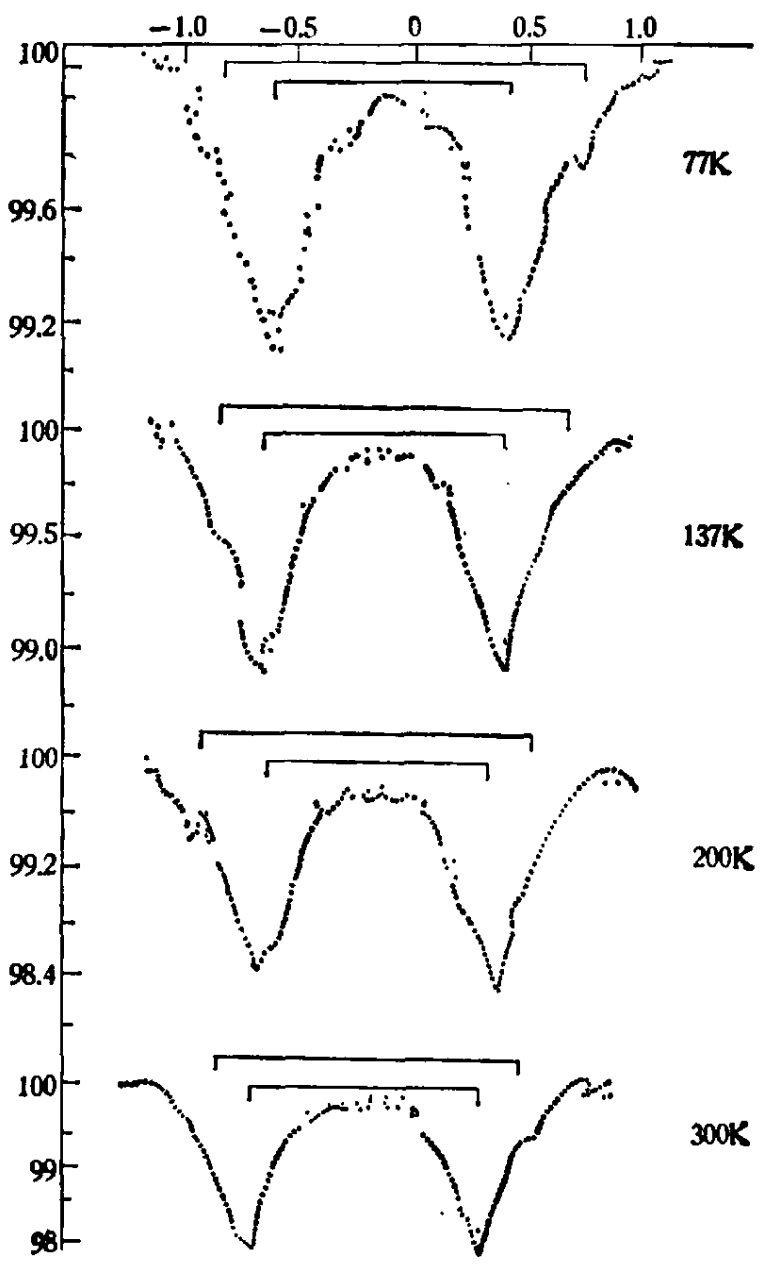

图 $4 \mathrm{LaFc}_{0 .} \mathrm{Ni}_{4.9}$ 的不同温度的 Mössbuer 吸收谱、放射源 ${ }^{37} \mathrm{Co} / \mathrm{Rh}$

型,大部分 (约 $90 \%$ ) $\mathrm{Fe}$ 原子分布于 $3 g$ 位,余下部份分布于 $2 C$ 位; 它们的同质异能位移 $\delta_{1.9}$ 
和四极分裂间卧 $\Delta E_{Q .5}$ 及 $f$ 因子为 $y$ 的函数, 但不同晶位的 $\delta_{1.5}$ 及 $\Delta E_{Q .5}$ 遥从不同的变 化规律. 在温度 $77-300 \mathrm{~K}$ 间, $\mathrm{LaFe}_{0.7} \mathrm{Ni}_{4.3}$ 的 $\Delta E_{Q .5}$ 随温度的下降而增大, 然而 $3 \mathrm{~g}$ 位和 $2 C$ 位的 $S_{I . S}$ 有蒴与此不同的温度关系. 由能量位移的温度关系, 估算 $\mathrm{LaFe}_{0.7} \mathrm{Ni}_{4.3}$ 的 Debye 温度为 $346 \mathrm{~K}$.

[1] Van Mal, H. H., Buschow, K. H. J. \& Miedema, A. R., J. Less-common Yet., 35 (1974), 65;

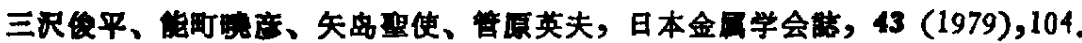

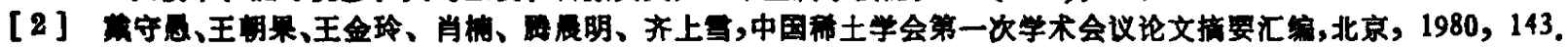

[8] a) Wagner, F. F. \& Wertmann, G., in Hydrogen in Metals I(Eds. Alefeld, G. \& Volkl, J.), BpringerVerleg, 1978, 131; b) Shenony, G. K., Dunlap, B. O., Viccaro, P. J. \& Niarchos, D., Hydrogen 8torage Matorials, Contribution to: Recont Chomioal Applications of Mossbaver Spectroscopy (Eds. Stevens, J. G \& Shenoy, G. K.), American Chemical Society Publication, 1982.

[ 4] Percheron-Guegon, A., Lartigue, O., Achard, J. C., Germi, P. \& Taset, F., J. Less-common Yet., 74(1980), 1.

[5] Ingalls, R., Van Der Woude, F. \& Sawatzky, G. A., in Mössbauer Isomer Shifts (Eds. Shenoy, G. K. Warmer, F. E.), North-Holland, 1978, 341.

[ 6] Williamson, D. L., in Mössbauer Isomer Shifts (Eds. Shenoy, G. K. \& Wagner, F. E.), Nörth-Holland, 1978, 317.

[ 7 ] Bhide, V. G., Mössbauer Effeot and Its Applications, New Dalhi, 1973, 102.

[8] Gutlich, P.. Link, R. \& Trautwein, A., Mäesbauer Spectroscopy and Transition Metal Chemistry, Springer-Verlar, 1978, 73.

[8] Wertheim, G. K., Buchanan, D. N. E. \& Guggenheim, H. J., Phys. Rev. B., 2(1870), 1392.

[10] a) Housley, R. M. \& Hess, F., Phys. Rev., 164 (1967), 164; b) Shrivastava, K. N., in Interne-

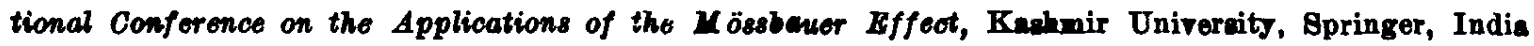
(abstracts), 1981, 225. 